Exercices Exercices de rhétorique

de rhétorique $15 \mid 2020$

Sur (et contre) les figures

\title{
Le soufre du paradoxe : Guyon relue par Godet des Marais (1695)
}

Agathe Mezzadri-Guedj

\section{CpenEdition}

1 Journals

Édition électronique

URL : http://journals.openedition.org/rhetorique/1041

DOI : $10.4000 /$ rhetorique. 1041

ISSN : 2270-6909

Éditeur

UGA Éditions/Université Grenoble Alpes

Édition imprimée

ISBN : 978-2-37747-248-2

Référence électronique

Agathe Mezzadri-Guedj, «L Le soufre du paradoxe : Guyon relue par Godet des Marais (1695) »,

Exercices de rhétorique [En ligne], 15 | 2020, mis en ligne le 07 novembre 2020, consulté le 03

décembre 2020. URL : http://journals.openedition.org/rhetorique/1041 ; DOI : https://doi.org/10.4000/ rhetorique. 1041

Ce document a été généré automatiquement le 3 décembre 2020.

\section{(c) () (ㅇ)}

Les contenus de la revue Exercices de rhétorique sont mis à disposition selon les termes de la Licence Creative Commons Attribution - Pas d'Utilisation Commerciale - Partage dans les Mêmes Conditions 4.0 International. 


\title{
Le soufre du paradoxe : Guyon relue par Godet des Marais (1695)
}

\author{
Agathe Mezzadri-Guedj
}

Dans De L'Autre Côté du miroir, la fringante Reine Blanche veut démontrer à Alice combien il est difficile d'adhérer à des paradoxes apparents ${ }^{1}$. Elle lui annonce, essoufflée par sa course, qu'elle a exactement cent un ans, cinq mois et un jour. Alice, éduquée selon les principes de la raison, rétorque qu'elle ne peut y "croire ». Croire serait donc consentir à ce que l'on préjuge comme vrai en l'absence de savoir positif : non pas une absence de jugement, mais un jugement fondé sur le critère du vraisemblable. La croyance devrait agir avec circonspection pour donner ou refuser son assentiment. Elle serait éminemment active, contrairement à la vision commune d'un mode de connaissance dégradé, crédule et paresseux.

2 Cependant, face à l'incrédulité d'Alice, la Reine lui propose de s'exercer régulièrement à croire « jusqu'à six choses impossibles » « avant d'avoir pris le petit déjeuner ». Voilà donc caricaturée l'hypothèse d'une croyance comme acte volontaire de l'esprit: il s'agirait simplement d'un " exercice » (vouloir croire) comme chercher à se faire rire soi-même... La croyance est-elle une disposition intime, en excès par rapport aux garanties objectives qu'offre son objet? Ce serait le modèle même de la foi comme sentiment s'emparant du sujet à travers la révélation plutôt que comme décision. $\mathrm{Ou}$ bien, peut-on décider de croire et avoir pour cela de bonnes raisons?

3 L'urgence de ces questions, loin d'être strictement épistémologique, engage la vie pratique et, dans le cas de notre corpus, la vie d'une femme. Prise dans la tourmente des condamnations opérées dès 1687, Madame Guyon est l'autrice de manuels pour s'exercer à ce qu'elle recherche activement et nomme "passiveté »: un amour pour Dieu si pur qu'il en accepterait la damnation éventuelle.

4 Ses contemporains, dont l'un des scribes est le père Godet des Marais, tenants d'une croyance raisonnée comme "donner son assentiment", reproduisent des morceaux choisis de ses ouvrages grâce au procédé bien connu de la citation sortie de son contexte. Autrement dit, même s'ils enracinent peut-être leur combat dans un « vouloir 
croire ", ils mènent la polémique sur le plan logique et font tout pour rendre les énoncés mystiques sulfureux, donc censurables.

Notons enfin que la stratégie exploite un substrat misogyne remontant au moins à Platon $^{2}$ et encore vivace chez nos philosophes des Lumières - pourtant souvent féministes avant la lettre ${ }^{3}$. Il s'agit de l'opposition entre parole virile, forte et raisonnable, et parole « efféminée ", molle et passionnée, voire hystérique. Il n'est donc pas indifférent que, lorsqu'en 1695, Paul Godet des Marais publie 63 extraits jugés condamnables de deux auteurs quiétistes, le père Lacombe et Madame Guyon, il recense quatre livres de Madame Guyon sur les cinq visés par son Ordonnance et, bien plus, il ne cite presque que... Madame Guyon (62 citations sur 63). La parole féminine lui fournit une matière source qu'il coupe, réécrit et colle à loisir pour faire jaillir, renforcer, parfois créer de toutes pièces, des figures sulfureuses du paradoxe.

Dans le détail du texte, nombre de ses arguments et critiques sont communs à Noailles, Harlay ou encore Bossuet. Ce qui est plus propre à Godet des Marais, c'est une méthode: la citation systématiquement décontextualisée d'une parole toujours féminine pour produire des figures sulfureuses du paradoxe et donc dignes d'être censurées. Cet article vise à recontextualiser dans la querelle du pur amour et à modéliser linguistiquement cette méthode.

\section{La chasse au paradoxe mystique (1687-1699)}

7 Entre 1687 et 1699, une véritable chasse aux mystiques a lieu en Europe. Les livres du prêtre Miguel de Molinos, du cardinal Petruci, du dominicain Tommaso Menghini, de la dominicaine Hippolyte de Jésus Rocaberti, du père Falconi, du père Lacombe, de Madame Guyon, de Malaval, de Bernières et de Fénelon sont mis à l'Index.

Ces ouvrages ont en commun de parachever une longue tradition d'opposition à la raison du paradoxe mystique depuis l'antique «science ignorante» du Pseudo-Denys. Déjà, en 1329, Maître Eckhart est condamné par la bulle papale In agro dominico. Au Xv siècle, Nicolas de Cues, auteur de l'oxymorique Docte ignorance, est attaqué par Jean Wenck au nom du principe de non-contradiction aristotélicien. Il en va de même pour les célèbres Thérèse d'Avila ${ }^{4}$ et Jean de la Croix pour le Xvi ${ }^{\mathrm{e}}$ siècle espagnol, Jean-Joseph Surin $^{5}$, pour qui le langage mystique est le langage de la contradiction ${ }^{6}$, François Malaval $^{7}$, puis Fénelon et Madame Guyon ${ }^{8}$ au XvII ${ }^{\mathrm{e}}$ siècle français. Aujourd'hui encore, pour le Dictionnaire de la mystique, le paradoxe « est à l'opposé de la pensée rationnelle » en vue de "s'approcher de l'inexprimable" ».

Cet aspect paradoxal de la parole mystique, du « galimatias ${ }^{10}$ » pour Bossuet, devient plus vivement critiqué à l'occasion de ce qu'il est convenu d'appeler la «querelle du pur amour ». Le Guide spirituel du prêtre espagnol Molinos se distingue des exercices ignaciens dans la mesure où, pour s'unir à Dieu, il s'agit de ne rien faire. Les rituels, ascèses et autres exercices d'imagination sont inutiles : c'est l'absence totale d'efforts (" quiétude ») qui permet l'union divine. Cette doctrine s'avère doublement paradoxale. Sémantiquement, elle unit passivité et action. Culturellement, elle s'oppose à la doxa de l'époque selon laquelle les œuvres - la charité notamment - et les exemples des saints sont nécessaires. Cette "quiétude» chez Molinos, "abandon» chez Malaval, "pur amour» chez Fénelon, prend la forme de l'oxymore (et donc du paradoxe) de l'« éloquent silence du Verbe dans l'âme ${ }^{11}$ » chez Guyon. 
10 Bien plus, pour Henri Bremond, cette figure du paradoxe mystique comme "merveille ", c'est-à-dire invraisemblance ${ }^{12}$, s'incarne dans la figure féminine de Madame Guyon pour expliquer l'extinction des mystiques :

Derrière Mme Acarie, derrière Mme Martin, je vois poindre une très haute, très séduisante, une fatale figure, Mme Guyon. Je ne compte pas accabler la noble femme qui voulut ressusciter sous Louis XIV les merveilles mystiques du temps de Henri IV et de Louis XIII. Je ne suis pas non plus de ceux qui tiennent Fénelon pour un génie malfaisant. Il est venu trop tard dans un siècle trop vieux et qui depuis trop longtemps riait des mystiques ${ }^{13}$.

Cette histoire littéraire mérite d'être nuancée. Avant même que ne fleurisse le topos romantique de la femme "fatale " réinvesti par Bremond, la décontextualisation et l'érotisation des paradoxes guyonniens sous l'angle de la folie hystérique sont opérées en 1694-1695 par l'évêque de Chartres et directeur de Madame de Maintenon : Paul Godet des Marais ${ }^{14}$.

\section{La mise à l'index (1688) et la condamnation épiscopale (1694-1695)}

11 Imprimé en mars 1685, le Moyen court de Madame Guyon est une vulgarisation abrégée des Torrents, œuvre de 1682 non publiée et qui sera révisée en 1703 . Le succès est immédiat, tout comme les réactions inquiètes des autorités religieuses ${ }^{15}$. Par sa Lettre pastorale contre le quiétisme, l'évêque de Genève provoque sa mise à l'index par le pape en 1688. À Saint-Cyr où le succès est retentissant, la visite canonique de Godet des Marais entraîne la confiscation des exemplaires en 1693. L'évêque parvient d'ailleurs à y bannir aussi les représentations théâtrales ${ }^{16}$. Il travaille enfin à la rédaction d'un Traité de l'Esprit de l'Institut des Filles de Saint-Louis. C'est donc dans le projet plus large d'éloigner les filles d'Ève des « désordres moraux » ou passions qui les guettent que des Marais rédige l'ordonnance qui provoque la prise en compte française de la condamnation papale contre les écrits de Madame Guyon.

En marge des entretiens d'Issy qui aboutissent à la condamnation des mystiques (dont Fénelon), il date du 16 avril 1695 et publie le $1^{\text {er }}$ mai 1695 « l'Ordonnance et instruction pastorale de Monseigneur l'évêque de Chartres" en vue de la "condamnation des livres intitulés Analysis orationis mentalis etc., Moyen court et très facile de faire oraison, etc., Règle des Associés à l'Enfance de Jésus etc. Le Cantique des Cantiques de Salomon, interprété selon le sens mystique etc., et d'un Manuscrit qui a pour titre Les Torrents ${ }^{17}$ ". Il s'agit apparemment de censurer un ouvrage du père Lacombe (Analysis) et quatre ouvrages de Madame Guyon, dont l'un est encore un manuscrit privé.

\section{Sous le pur amour, un Éros socratique dans le corps d'une femme}

13 Avant d'étudier la méthode de Des Marais, il convient de reconnaître le potentiel érotique de l'écriture de celle qui "cherch[e] Dieu en-dedans et non au-dehors », et qu'Emerson relit pour se réchauffer du froid de la Nouvelle-Angleterre ${ }^{18}$, et de s'entendre sur le qualificatif érotique. L'éros guyonnien se rattache moins à la figure d'Ève qu'à celle du daïmôn socratique, cette force intérieure christianisée depuis Marsile Ficin et qui n'est ni l'Éros primordial de Phèdre, ni l'Éros-Cupidon d'Agathon, encore moins la bête compulsionnelle d'Aristophane.

14 Ceci étant dit, la mystique aggrave, si l'on peut dire, son ethos féminin d'autrice en évoquant sa foi comme un feu intérieur brûlant ${ }^{19}$, souvent rapide et violent ${ }^{20}$, ou sa 
rédaction du Cantique des cantiques en un jour et demi ${ }^{21}$. Bernard Forthomme et Jad Hatem s'intéressent ainsi à son "désir de la vitesse », pour "tomber en Dieu ${ }^{22}$ ». En outre, l'enthousiasme se double d'une féminité assumée à travers les figures bibliques de Rebecca, la femme de Lot, Debora, Ruth, Esther, ou encore Marie ${ }^{23}$. Cette force intérieure, ce Dieu au-dedans hérité du daïmôn ressemble donc, par endroits, au jeune fougueux Cupidon dans une enveloppe féminine.

Aujourd'hui encore, des éditeurs choisissent de lire "hystériquement » plutôt qu' « historiquement » dans le passage ci-dessous des Torrents :

Il me semble que l'âme de ce premier degré, même dans les plus avancés, a une certaine habitude à cacher ses défauts et à elle et aux autres. Elle trouve des excuses et des prétextes. Elle ne les dit jamais ingénument: non par volonté, mais par un certain amour de sa propre excellence, par une dissimulation habituelle sous laquelle elle se cache. Elle n'a pas tant de paix dans ses misères : au contraire, elle se sent affligée extraordinairement. Elle a certain empressement de s'en purifier. Elle le dit historiquement ${ }^{24}$.

Selon Morali ${ }^{25}$, bien que la mystique ait écrit "historiquement» dans toutes ses versions, il semble plus logique de lire «hystériquement» en raison d'un ajout manuscrit dans une seule copie, mais surtout parce que cela correspond à la représentation de Madame Guyon en hystérique. On peut opposer à Morali que l'adverbe « historiquement » convient parfaitement à cette âme qui se drape de dignité pour revendiquer pompeusement ses efforts. Bien plus, on sait que l'extase infinie de Madame Guyon est en réalité plus proche de Thanatos que d'Éros. Il n'est en aucun cas question de laisser place à un érotisme du corps dans l'exercice des contritions et la nudité est un mouvement de "mortification ${ }^{26} »$ :

Il y a une manière d'aller à Dieu par voie d'élévation au-dessus de soi, et celle-là est accompagnée d'extases et de ravissements. Il y a une autre manière de sortir de soi par voie d'anéantissement et de nudité, et celle-là n'a point d'extase : c'est une voie toute de mort, et par cette mort l'âme sort de soi et passe dans une extase permanente en son Divin objet [...] non en se multipliant au dehors et retournant d'où l'on est venu, mais en [...] s'outrepassant pour entrer en Dieu ${ }^{27}$.

L'hypothèse hystérique des éditeurs actuels - étymologiquement la maladie de celles qui ont un utérus, et que tous les dictionnaires du siècle rapprochent de la passion-, accrédite l'idée que l'ajout de paradoxes sulfureux aux écrits, certes peu classiques, de Madame Guyon en a gauchi la réception. Pour produire cette déformation de la parole guyonnienne, la figure du paradoxe en a été l'outil idéal. La parole féminine hystérique aurait des «affections", dont les prolongements sont moraux et esthétiques en cette fin de siècle de l'ordre et de la raison.

\section{Les Torrents de paradoxes contre le ruisseau classique}

17 Dans le contexte de la querelle du pur amour et à l'époque du style coulant comme un ruisseau de Boileau, le paradoxe est déconsidéré, au point de constituer une sorte d'insulte dans les querelles théologiques. Il est même rarement considéré comme une figure ou une catégorie logique aux siècles classiques ${ }^{28}$.

Le mot paradoxe entre en langue française avec un caractère bifrons aux alentours de 1370. Défini comme ce qui est " au-dessus de ce qu'on peut croire ${ }^{29}$ ", son sens passe de l'extraordinaire vérité à l'erreur absurde, selon que la doxa est considérée comme vraie ou fausse. La consultation des dictionnaires classiques ne dément pas cette élasticité ${ }^{30}$. Toutefois, les emplois en contexte théologique révèlent une prégnance négative et une 
proximité entre paradoxe, sophisme, pétition de principe et faux raisonnement au tournant des XVII et XVIII ${ }^{\text {e }}$ siècles. Paradoxe et contradiction sont loin d'être distingués et le sème de /vérité/ n'est presque plus attribué au paradoxe. Dans les querelles théologiques du Grand siècle, il scelle le ridicule d'un discours contradictoire ${ }^{31}$ selon le mécanisme bien connu de l'argument d'incompatibilité ${ }^{32}$ auquel se réfère la reine de Lewis Carroll.

En outre et malgré l'emploi du paradoxe par les moralistes comme La Rochefoucauld ou La Bruyère, la figure du paradoxe disparaît presque totalement des rhétoriques classiques et ce, dès Scaliger en $1561^{33}$. Ni Louis de Cressolles ${ }^{34}$, ni René Bary ${ }^{35}$, ni Louis Bourdaloue $^{36}$, ni Bernard Lamy ${ }^{37}$, ni l'abbé Breton ${ }^{38}$ ni Joseph de Jouvancy (qui fait mention de l'« éloge ironique " parmi ses figures de pensée ${ }^{39}$ ) ne l'évoquent une seule fois dans leurs manuels. Le père Rapin ${ }^{40}$ et Vaugelas n'en parlent pas davantage ${ }^{41}$ et la situation se perpétue - bien que moins perceptiblement - au XVIII ${ }^{\mathrm{e}}$ siècle. Seul Crevier lui donne véritablement ses lettres de noblesse en $1765^{42}$. Du côté des poétiques, le silence, à notre connaissance, est total.

La première des deux apparitions du paradoxe comme figure dans une rhétorique du siècle se trouve chez Nicolas Caussin. Est recopiée la définition antique du paradoxe comme figure de mots de Julius Rufinianus, et que l'on qualifierait aujourd'hui de par'hyponoian :

Paradoxon ou hypomonè, suspension ou surprise. Cette figure retarde le sens, puis lui en substitue un autre, contrairement à ce qu'attend l'auditeur, que ce soit par ajout de sens ou retranchement, et, par conséquent, on l'appelle suspension ou surprise. Ainsi, Cicéron, dans son Plaidoyer pour Ligarius : « Il en est écarté ; ce n'est pas auprès de César qu'il se rend, on aurait cru à de la colère; ni à Rome, on aurait cru à un renoncement; ni dans quelque province, on aurait cru à une condamnation de la cause qu'il avait suivie $"$, etc. ${ }^{43}[\ldots]$.

Un autre ouvrage mystérieux fait grand cas du paradoxe : La Clavicule, appelée aussi $L a$ Clef universelle car il donnerait accès à tous les arts, de Raymond Lulle. Cet ouvrage attribué au franciscain et mystique originaire de Majorque circule dans la deuxième moitié du XvII ${ }^{\mathrm{e}}$ siècle grâce aux sieurs Jacob et Colletet qui en sont vraisemblablement les auteurs. Il s'agit d'un relevé de « toutes » les figures de rhétorique :

Le Paradoxe est fort agréable, et s'acquiert bien souvent une grande attention. Cette Figure consiste à faire des Propositions extraordinaires, qui semblent d'abord contraires à l'intelligence commune, et qui ne sont véritables que selon un sens caché, et fort éminent. On y promet de traiter de choses grandes, nouvelles, et extraordinaires, et la plupart des hommes écoute cela volontiers. Ainsi je proposerai de faire connaître, Que personne n'est offensé que par soi-même, Que la Vertu seule est capable de rendre un homme heureux, et que les Sujets, qui sont tant estimés du vulgaire en cette vie, ne sont pas de vrais Biens. Ce sont tous Paradoxes, et à dire vrai, ces Propositions, qui sont éloignées des sentiments communs, suspendent les Esprits, et leur donnent beaucoup de satisfaction : quelques-uns préparent l'esprit du Lecteur à les entendre ${ }^{44}[. .$.$] .$

Nous retrouvons dans cette définition notre sens actuel de figure à double détente : l'apparence de la fausseté d'abord, l'effet de vérité ensuite ${ }^{45}$.

Chez Madame Guyon, on rencontre une occurrence du mot paradoxe qui n'est pas étrangère à cette approche pragmatique de la double détente, tout en se rapprochant de la définition qu'en donne Bouhours à travers Eudoxe dans La manière de bien penser : "faux et vrai tout ensemble", mais "faux» si l'on ne regarde que "l'écorce de la pensée $e^{46} »$. Le terme, présent dans sa Vie par elle-même, ne s'inscrit pas directement dans une terminologie rhétorique, et permet de décrire les âmes mystiques : 
Ces personnes sont un paradoxe et à leurs yeux et aux yeux de tous ceux qui les voient, car l'on ne voit qu'une écorce grossière, bien que pourtant il en sorte souvent une moelle divine. En sorte que ceux qui en veulent juger par les yeux de la raison, ne savent pas par où s'y prendre. $O$ divine sagesse, ô science savoureuse, vous coulez incessamment de la bouche et du cœur de ces âmes comme une source divine qui communique la vie à une infinité de branches, quoiqu'on ne voie qu'une écorce grossière et toute moussue ${ }^{47}$.

Madame Guyon remotive donc l'« écorce » stylistique de Bouhours pour qualifier, non ses propres écrits, mais la scission essentielle du sujet mystique, telle l'âme platonicienne du Phèdre. Elle explique que l'apparence grossière de ces personnes est ce qui les rend encore dépendantes du monde, ce qu'elle appelle aussi être un " animal» ou une « bête ». Leur âme divine, au contraire, est « libre ».

Par l'emploi d'une figure minorée dans les rhétoriques classiques, Madame Guyon place sa parole dans la continuité d'une logique minoritaire: celle de Johannes Clauberg (1622-1665), seule à mentionner le paradoxe - les précartésiens Jean de Champaynac ${ }^{48}$ et Scipion Dupleix ${ }^{49}$, ni même Arnout Geulincx ${ }^{50}$ ne le mentionnent, et la logique la plus célèbre, celle de Port-Royal, le condamne implicitement au motif qu'il est indécent de s'opposer brutalement au sens commun. Dans la logique dite cartésienne de Clauberg, le paradoxe apparaît deux fois pour opposer le dialecticien, entendu au sens de raisonneur consensuel, à l'authentique philosophe qui ose combattre les fausses vérités :

Le dialecticien se sert des contingents et des probables pour débattre de certains sujets de façon catégorique ou sinon dubitative. Le philosophe se sert des nécessaires et souvent de paradoxes : l'un affirme avec réserve [verecunde affirmat] pour instruire des auditeurs dociles, et l'autre assure plus hardiment [confidentius asserit] pour réfuter les opiniâtres ${ }^{51}$.

À ce choc produit par le paradoxe qui servirait à emporter l'adhésion d'un auditoire plus réfractaire et serait plus l'apanage du "philosophe » que du prudent " dialecticien », s'ajoute le fait que le paradoxe serait rendu nécessaire par la difficulté de l'objet du discours et ne pourrait convaincre que peu d'auditeurs :

[...] dans les interprétations complexes, il énonce fréquemment des paradoxes, c'est-à-dire ce qui va à l'encontre de l'opinion du vulgaire, para doxan. Et parce qu'il sait que la plupart des hommes observent plus facilement l'apparence de la vérité [speciem veritatis] que la vérité même, et qu'ils portent plus souvent des jugements défectueux que corrects, aussi se contentant de peu de juges, il fuit sciemment la multitude, sans rechercher ni attendre l'applaudissement et les suffrages de la foule. C'est tout de même le fait du bon maître, dans l'enseignement des disciplines, que de rendre probables à ses disciples et aux lecteurs soucieux du vrai, ce qui paraît des paradoxes aux autres, dans la mesure où c'est possible ${ }^{52}$.

Réservé à un petit lot d'initiés, le paradoxe ne peut convaincre d'emblée une foule, en particulier à l'époque de la clarté et des ruisseaux de Boileau. Le grand nombre serait trop attaché à « l'apparence de la vérité » et c'est cet attachement à la vraisemblance et au consensus contre la « merveille » des paradoxes qu'exploite Godet des Marais.

\section{La figure du paradoxe : contre-vérité apparente et amputation du contexte}

23 Étymologiquement, le paradoxe s'oppose aux deux dimensions de la doxa : rationnelle (logique commune ou bon sens) et sociale (opinion commune). Dans le sens le plus employé par les philosophes, la doxa est l'opinion commune, au sens de « courante » ou «vulgaire». Parmi les disciples de Socrate, elle prend l'apparence d'une évidence, 
malgré l'absence de clarté et de véritables preuves $^{53}$ : les paradoxes socratiques dépassent la vérité apparente de l'opinion commune par le raisonnement logique. Le second sens de doxa est le "sens commun ${ }^{54}$ ", c'est-à-dire précisément la logique, parfois appelée «bon sens ». Cette doxa-logique peut aussi être considérée comme une vérité apparente au sens où l'on y accède par le biais de l'évidence, d'une intuition ou vision immédiates. C'est le cas des «axiomes, c'est-à-dire les propositions claires et évidentes par elles-mêmes ${ }^{55}$ ». C'est pourquoi, sur le plan référentiel, le paradoxe se définit précisément comme la "contradiction d'une vérité apparente ${ }^{56}$ ». Cette définition référentielle s'adosse à la définition formelle de Marion Carel et d'Oswald Ducrot. Un paradoxe est :

Un enchaînement d'énoncés, un énoncé ou un mot, qui réalisent, de façon interne, un aspect argumentatif A DONC (resp. POURTANT) B, alors que la signification « intrinsèque » de $\mathrm{A}$ ne comporte pas cet aspect et, en outre, comporte l'aspect $\mathrm{A}$ POURTANT (resp. DONC) B ${ }^{57}$.

La notion de vérité apparente est convoquée dans le syntagme "signification "intrinsèque" " pour être contredite « de façon interne » dans le processus paradoxal.

La deuxième caractéristique du paradoxe permettant de comprendre le travail de sape de Godet des Marais est que tout paradoxe repose sur une amputation de son contexte discursif, travail auquel se livre l'évêque de Chartres afin de renforcer les paradoxes de Madame Guyon et d'en créer de toutes pièces.

Le lien consubstantiel entre paradoxe et contextualisation apparaît déjà chez Aristote. Dans le passage de La Rhétorique consacré aux enthymèmes, le philosophe suggère que c'est une abstraction du contexte qui les rend paradoxaux :

[...] par exemple, une femme ayant renversé sous elle son propre fils à force de l'embrasser, on crut qu'elle faisait l'amour avec le jeune homme; la cause expliquée, la suspicion disparut ${ }^{58}$.

Sachant que l'enthymème est un syllogisme tronqué parce que la majeure, doxale, est connue de tous, le paradoxe mis en valeur ici apparaît comme son symétrique inverse : une proposition, inconnue de tous, est à rétablir. L'amputation d'un élément de contexte est la caractéristique nécessaire du véritable paradoxe. C'est ce que pratique Godet des Marais lorsqu'il cite Madame Guyon : les paradoxes sont alors amplifiés, et même, rendus sulfureux.

\section{Débusquer Ève}

\section{Le 27 novembre 1695 Madame Guyon écrit «À la petite duchesse » :}

[...] je dois un aveu de la vérité au public. Je dirai donc que je ne reconnais point l'écrit des Torrents dans la lettre pastorale de $\mathrm{M}$. de Chartres, que je le vois seulement travesti, qu'il est absolument méconnaissable, ceux qui l'ont transcrit avec une fin malicieuse ayant ajouté des endroits et tronqué d'autres qui le rendent tout à fait différent de lui-même [...].

Mais pour autant de choses qu'on m'impute par des sens si violents qu'on donne à mes écrits, qu'il serait très aisé de justifier et d'en faire voir la pureté et l'innocence, je déclare qu'on m'impute des pensées, qu'on donne des tours auxquels je n'ai jamais pensé.

[...] Le soin qu'on a pris de tronquer les passages, d'ajouter à d'autres, marque assez le peu de bonne foi qu'on a conservé de tout cela ${ }^{59}$.

De fait, dans l'Ordonnance de Godet des Marais, il n'y a pas que les Torrents qui sont remaniés : les 63 extraits jugés condamnables de livres quiétistes comptent 62 extraits des livres de Madame Guyon, tous coupés, augmentés, parfois rapprochés de passages 
situés à plusieurs pages d'écart ${ }^{60}$, comme s'il s'agissait du même paragraphe ${ }^{61}$. À une exception près, les sauts et omissions ne sont jamais signalés dans les extraits numérotés de I à LXIII. Dans le détail, il s'agit de 38 citations des Torrents, 14 citations du Moyen Court, 8 citations du Cantique des Cantiques de Salomon interprété selon le sens mystique, 2 citations des Règles des Associés et une seule de l'Analysis du Père Lacombe. À une exception près, Godet des Marais ne cite donc que Madame Guyon et, en grande partie un manuscrit à caractère totalement privé, non publié, qu'il s'est procuré par des moyens détournés: les Torrents. La condamnation du langage mystique repose sur l'analyse de la parole féminine en priorité (62 citations sur 63) et sur son versant intime (38 citations des Torrents sur 63), comme s'il s'agissait de faire surgir Ève de l'ombre pour démonter son discours.

\section{La déformation des paradoxes en hérésie sulfureuse}

27 Par son mécanisme à double détente, la figure du paradoxe se caractérise par son ambivalence. L'impression d'erreur pour laisser place à une vérité plus grande peut être figée en erreur, voire en hérésie, par de très légères déformations sémantiques ou graphiques. C'est ce à quoi s'emploie l'évêque. Par des coupes et inversions, il dénature sémantiquement les paradoxes de Madame Guyon et transforme le paradoxe de l'oraison de quiétude en hérésie.

Dans le détail des deux ouvrages les plus cités par des Marais, ce sont les chapitres explicitement dédiés au paradoxe de l'oraison de quiétude - " passiveté », " repos » et "abandon" pour le Moyen court, "Voie passive en foi" pour les Torrents - qui fournissent matière à son inventaire ${ }^{62}$. Face à la doxa - culturelle - de l'époque (étudiée par Vincent Carraud $\left.{ }^{63}\right)$, le paradoxe peut se formuler ainsi : je réalise des œuvres DONC j'ai droit au salut à quoi Guyon répond je ne réalise pas d'œuvres POURTANT j'ai droit au salut.

On peut supposer que le reste n'a été consulté que rapidement tout au plus: l'ordonnance qui se présente comme une analyse point par point de cinq ouvrages quiétistes n'est en réalité qu'une rationalisation a posteriori d'une condamnation déjà prononcée et qu'il s'agit de rendre légitime en chargeant l'accusation de paradoxes. C'est pourquoi, Godet des Marais transforme, par des coupes, interversions et réagencements, tout ce qui pourrait contextualiser le paradoxe sémantique et culturel de l'oraison de quiétude en hérésies et désordres moraux.

L'exemple le plus évident concerne le chapitre XIII du Moyen Court intitulé «Du repos devant Dieu » qui, à l'origine, ancre le discours de Madame Guyon dans une morale chrétienne active (oraison permanente, présence de Dieu, refus du monde: «l'extérieur », pratique des «vertus » et préférence pour les actions «bon[nes] » sur les « mauvais[es]») :

1. L'âme étant arrivée ici n'a plus besoin d'autre préparation que de son repos. Car c'est ici que la présence de Dieu durant le jour, qui est le grand fruit de l'oraison, ou plutôt la continuation de l'oraison même, commence d'être infuse et presque continuelle. L'âme jouit dans son fond d'un bonheur inestimable. Elle trouve que Dieu est plus en elle qu'elle-même. Elle n'a qu'une seule chose à faire pour Le trouver, qui est de s'enfoncer en elle-même. Sitôt qu'elle ferme les yeux, elle se trouve prise et mise en oraison. Elle est étonnée d'un si grand bien, et il se fait audedans d'elle une conversation que l'extérieur n'interrompt point.

2. On peut dire de cette manière d'oraison ce qui est dit de la Sagesse, que tous biens sont venus avec elle. Car les vertus coulent agréablement en cette âme qui les pratique d'une manière si aisée qu'elles semblent lui être naturelles. Elle a un 
germe de vie et de fécondité qui lui donne de la facilité pour tout ce qui est bon, et de l'insensibilité pour tout ce qui est mauvais.

3. Qu'elle demeure donc fidèle en cet état, et qu'elle se donne bien en garde de chercher d'autre disposition, quelle qu'elle soit, que son simple repos, soit pour la confession ou communion, action ou oraison ${ }^{64}$.

L'extrait XXXII de l'Ordonnance de Godet des Marais déforme ce paradoxe en tronquant tout ce qui apparaissait à la forme affirmative dans le Moyen Court, y compris l'injonction à conserver une attitude génératrice de vertu ( $Q u$ 'elle demeure donc fidèle en cet état»). Par la succession de deux négations exceptives, il invente une morale du refus et de l'inaction :

[\$1 début :] L’âme étant arrivée ici n’a plus besoin d'autre préparation que de son repos. [Omission exceptionnellement signalée par des points de suspension; §3 :] Qu'elle se donne bien en garde de chercher d'autre disposition, quelle qu'elle soit, que son simple repos, soit pour la confession ou communion, action ou oraison ${ }^{65}$.

La coupure des paragraphes 1,2 et 3 , retire au paradoxe du « repos » son contexte profondément positif. Bien plus, stylistiquement, la coupure fait s'enchaîner deux négations exceptives qui ne se suivaient pas chez Madame Guyon, rendant le paradoxe plus vif et donc plus sulfureux : "n'a plus besoin d'autre préparation que », « en garde de chercher d'autre disposition [...] que». Dans la négation exceptive, ne plonge l'énoncé dans la négation tandis que l'adverbe que le réoriente vers une visée positivante selon la théorie guillaumienne ${ }^{66}$. De ce fait, le regard du lecteur est attiré vers ce qui se trouve après que: le fameux et paradoxal « repos ». En outre, le rythme de la phrase place un point d'acmé au niveau de l'adverbe que, isolant le terme même de repos qui était justifié par le contexte dans le texte original. Décontextualisant l'idée de « repos » de celle de " vertu », la coupe de Godet des Marais le rend hérétique.

C'est bien pour les dresser contre les vertus théologales que Godet des Marais rend les paradoxes guyonniens sulfureux. Sa stratégie est transparente : il s'agit de faire passer Madame Guyon pour une hérétique en lui faisant nier les vertus théologales. Fénelon l'a bien compris. Il écrit dans l'une de ses multiples réponses : « Bossuet dit que Madame Guyon a déclaré dans sa Vie, que les vertus n'étaient plus pour elle, etc. et que j'ai adopté ses paroles en disant qu'on ne veut plus les vertus comme vertus, et que pour les rabaisser j'ai fait violence à tant de passages de saint François de Sales qu'il fallait entendre plus simplement avec le saint ${ }^{67}$ ».

Des trois vertus théologales ${ }^{68}$, la foi est la seule à laquelle les adversaires de la mystique ne peuvent s'attaquer. Il reste l'espérance et la charité. La foi de Madame Guyon serait alors tellement hystérique qu'elle en viendrait à cannibaliser les deux autres vertus que sont l'espérance, puis la charité.

\section{Contre l'espérance : relatives essentielles supprimées, graphèmes inversés}

Dans les Torrents, Madame Guyon écrit: «si on a alors un Directeur qui n'est pas expérimenté et qui oblige cette âme à prier [vocalement], outre qu'il lui fait souffrir une gêne très grande, il lui fait un tort irréparable ${ }^{69} »$. Dans son Ordonnance, numéroté XXI, l'extrait de Godet des Marais est le suivant : «Si un directeur oblige cette âme à prier, il lui fait un tort irréparable ${ }^{70} »$. Godet des Marais supprime la relative adjective " qui n'est pas expérimenté » énonçant une propriété qui dans l'acte de communication accompli apparaît comme essentielle (par opposition aux relatives adjectives 
accidentelles ou accessoires dont la suppression ne remet pas en cause l'information fondamentale de l'énoncé ${ }^{71}$ ). Supprimer l'hypothèse d'une défaillance due à l'inexpérience du directeur de conscience change radicalement l'idée qu'il puisse causer de la "souffrance " à l'âme dont il a la charge. Dans la suite de la phrase, supprimer "outre qu'il lui fait souffrir une gêne très grande " intensifie l'effet de surprise dû au paradoxe nouvellement créé par Godet des Marais. Sans aucun lien avec l'idée originale selon laquelle l'inexpérience pourrait mener à des accompagnements spirituels dommageables, la citation de l'évêque de Chartres fait dire à la mystique que prier cause du tort: "Si un directeur oblige cette âme à prier, il lui fait un tort irréparable ». L'énoncé est en tous points contraire au dogme et, en ce sens, hérétique. Sachant que les Torrents constituent un manuscrit privé jusqu'au début du XVIII ${ }^{\mathrm{e}}$ siècle, on comprend aisément comment cette diffusion de seconde main a pu entraîner l'emprisonnement de sa supposée autrice.

La suite de ce même passage est exploitée au numéro XXXIII de l'Ordonnance de Godet des Marais: "C'est alors qu'elle commence à ne pouvoir gagner les indulgences; et l'amour ne lui permet pas de vouloir abréger ses peines ${ }^{72}$ ", écrit-il de l'âme en disant citer Guyon. Or, Madame Guyon écrit : «C'est alors que l'âme a un désir de souffrir si véhément qu'il la fait languir et mourir. Elle voudrait payer pour les péchés de tout le monde et satisfaire à Dieu. C'est alors qu'elle commence à ne pouvoir gagner les indulgences et l'amour ne lui permet pas de vouloir abréger les peines ». Dans le texte de Madame Guyon, en rétablissant la phrase précédente, on voit bien que l'âme cherche, telle Jésus-Christ, à assumer « les peines » d'autrui et que cette étape de la vie mystique, particulièrement difficile, réclame de la patience. Or, le déterminant possessif «ses » en lieu et place du défini «les » suggère, chez des Marais, que l'âme cherche à abréger ses propres souffrances. Godet des Marais transforme, par un simple graphème ( $s$ » en lieu et place de « 1 ») la peine du monde en suggestion, sinon du suicide, du moins de la perte de la vertu théologale qu'est l'espérance. Cette interprétation est étayée par l'extrait dit XXXIV de l'Ordonnance (« Il n’y a plus rien pour elle, plus de règlement plus d'austérités ; tous les sens et les puissances sont dans le désordre $\left.{ }^{73} »\right)$ qui dit se référer aux Torrents sans qu'il soit possible d'en retrouver la trace.

\section{Contre la charité : des sentences paradoxales sans " épilogue »}

Le paragraphe 2 du chapitre VI (« De l'abandon ») du Moyen Court, de Madame Guyon, se présente à l'origine ainsi :

2. L'abandon est ce qu'il y a de conséquence dans toute la voie, et c'est la clef de tout l'intérieur. Qui sait bien s'abandonner, sera bientôt parfait. Il faut donc se tenir ferme à l'abandon sans écouter le raisonnement ni la réflexion. Une grande foi fait un grand abandon. Il faut s'en fier à Dieu, espérant contre toute espérance $e^{74}$.

3. L'abandon est un dépouillement de tout soin de nous-mêmes, pour nous laisser entièrement à la conduite de Dieu. Tous les chrétiens sont exhortés à s'abandonner. Car c'est à tous qu'il est dit: Ne soyez pas en souci pour le lendemain car votre Père céleste sait tout ce qui vous est nécessaire ${ }^{75}$. Pensez à Lui dans toutes vos voies et Il conduira Lui-même vos pas ${ }^{76}$. Exposez vos ceuvres au Seigneur et Il fera réussir vos pensées ${ }^{77}$. Remettez au Seigneur toute votre conduite et espérez en Lui, et il agira Lui-même $e^{78}$.

4. Pour la pratique elle doit être de perdre sans cesse toute volonté propre dans la volonté de Dieu, renoncer à toutes inclinations particulières, quelque bonnes qu'elles paraissent, sitôt qu'on les sent naître, pour se mettre dans l'indifférence et 
ne vouloir que ce que Dieu a voulu dès son éternité. Être indifférent à toutes choses, soit pour le corps soit pour l'âme, pour les biens temporels et éternels. Laisser le passé dans l'oubli, l'avenir à la Providence, et donner le présent à Dieu. Nous contenter du moment actuel qui nous apporte avec soi l'ordre éternel de Dieu sur nous, et qui nous est une déclaration autant infaillible de la volonté de Dieu qu'elle est commune et inévitable pour tous ${ }^{79}$.

Dans ces paradoxes, on note un travail rythmique de la mystique autour de toutes les sentences, qu'elles soient paradoxales ou doxiques. Le rythme impair, qui frôle l'alexandrin tout en évitant le vers blanc, donne à mémoriser tout autant la notion de repos ici nommé «abandon», que celles de «foi » et d'espérance : "Qui sait bien s'abandonner, sera bientôt parfait » [7/6] / " Il faut donc se tenir ferme à l'abandon sans écouter le raisonnement ni la réflexion. Une grande foi fait un grand abandon [5/1/5]. Il faut s'en fier à Dieu, espérant contre toute espérance ${ }^{80}[3 / 2 / 3 / 3]$ ». Dans le contexte (supprimé par Godet des Marais), Guyon suggère que l'abandon prôné est tout le contraire d'une absence de foi («Une grande foi fait un grand abandon»), ou d'espérance (« espérant contre toute espérance ${ }^{81} »$.)

Dans son Ordonnance, où Godet des Marais réécrit le paragraphe 2 citant ce passage, il renforce la sentence paradoxale sur l'«abandon» en lui retirant son contexte de sentences, tout aussi mémorisables, mais plus doxiques, autour de la «foi » et de l'« espérance ». Il écrit :

[§ 2 :] Qui sait bien s'abandonner, sera bientôt parfait. [large coupure sans indication ; § 4 :] Pour la pratique elle doit être de perdre sans cesse toute volonté propre [omission de «dans la volonté de Dieu »], de renoncer à toutes inclinations particulières, quelque bonnes qu'elles paraissent, sitôt qu'on les sent naître, pour se mettre dans l'indifférence et ne vouloir que ce que Dieu a voulu dès son éternité. Être indifférent à toutes choses, soit pour le corps soit pour l'âme, pour les biens temporels et éternels. Laisser le passé dans l'oubli, l'avenir à la Providence, et donner le présent à Dieu. Nous contenter du moment actuel qui nous apporte avec soi l'ordre éternel de Dieu sur nous, et qui nous est une déclaration autant infaillible de la volonté de Dieu qu'elle est commune et inévitable pour tous ${ }^{82}$.

La création d'une coupure juste après la sentence paradoxale fait disparaître les sentences consacrées aux deux vertus théologales de la foi et de l'espérance selon des rythmes tout aussi réguliers. Le paragraphe 3, entièrement coupé, est tout aussi essentiel. Godet des Marais supprime les passages doxiques consacrés à la foi et à l'espérance qui permettent l'abandon $(\$ 2)$ et la définition de l'abandon comme charité voire pur amour (§3).

De ce fait, l'indifférence héritée d'Ignace de Loyola devient inaudible puisqu'elle se réduit à une sentence paradoxale sans ce qu'Aristote nomme "l'épilogue " des maximes, explication nécessaire «quand elles expriment quelque chose de paradoxal ou de contesté ${ }^{83}$ ", et la Rhétorique à Alexandre les "justifications ", qui permettent " d'éviter à la fois le parler creux et la défiance ${ }^{84}$ ». En supprimant l'épilogue, Godet des Marais fait passer la sentence guyonnienne de " contre-vérité apparente » à " contrevérité », de paradoxe à contradiction insurmontable.

Or, si le pur amour guyonnien renoue sans nul doute avec un Éros socratique d'avant le De Amore de Marsile Ficin, il ne s'oppose nulle part explicitement à Agapè - la charité. Il n'est jamais non plus aussi érotique que l'évêque de Chartres nous invite à le penser. 


\section{Transformer la foi en passion : la suppression de l'expression de la cause}

" [I]ls ouvrent par leurs maximes la porte à un libertinage sans bornes ", prévient Paul Godet des Marais à la page 4 de son Ordonnance ${ }^{85}$, en dénonçant ainsi la tentation du «libertinage sans bornes » de tous les mystiques (même s'il ne cite de fait que Madame Guyon). Cette sentence fonctionne comme un pacte d'écriture. Les 63 « maximes » qui vont suivre entraîneraient une liberté de mœurs qui n'est que désordre moral. Ce point de vue qui opère toutes les coupes, va même jusqu'à érotiser ce qui, pourtant, ne l'était pas sous cet angle dans le texte original. Sans aller jusqu'au libertinage du siècle à venir, Godet des Marais accuse Guyon de se soumettre aux passions. Un passage des Torrents illustre ce mécanisme ; Madame Guyon y écrit :

L'âme étant appliquée directement à l'austérité et au-dehors, elle est toute tournée de ce côté-là, de sorte qu'elle met les sens en vigueur, loin de les amortir.

Car les sens ne peuvent tirer de vigueur que de l'application de l'âme, qui leur communique d'autant plus de vie qu'elle est plus en eux. Cette vie des sens émeut et irrite la passion, loin de l'éteindre. Les austérités peuvent bien affaiblir le corps, mais jamais émousser entièrement la pointe des sens ni leur vigueur, par la raison que je viens de dire.

Une seule chose le peut faire, qui est que l'âme par le moyen du recueillement se tourne toute au-dedans d'elle pour s'occuper de Dieu qui y est présent ${ }^{86}$.

Dans l'Ordonnance de Godet des Marais, l'extrait XXXV se présente ainsi :

L'âme étant appliquée directement à l'austérité et au-dehors, elle est toute tournée de ce côté-là, de sorte qu'elle met les sens en vigueur, loin de les amortir. [omission] Les austérités peuvent bien affaiblir, mais jamais émousser entièrement la pointe des sens ni leur vigueur, par la raison que je viens de dire.

Une seule chose le peut faire, qui est que l'âme par le moyen du recueillement se tourne toute au-dedans d'elle pour s'occuper de Dieu qui y est présent ${ }^{87}$.

Ce que supprime Godet des Marais, c'est une indépendante à valeur causale introduite par la conjonction de coordination car avec laquelle, selon Anscombre, "l'énonciation s'appuie sur le lien causal, mais ne l'introduit pas $^{88}{ }^{8}$. L'indépendante n'insiste pas autant sur la démarche logique que les subordonnants parce que et puisque. Deux conséquences en découlent : contrairement à Fénelon, par exemple, mais aussi parce qu'il s'agit encore ici d'un manuscrit privé, Madame Guyon est moins soucieuse de mettre en scène l'architecture logique de son propos. Partant, Godet des Marais se saisit de cette négligence pour faire disparaître l'expression de la cause en «car » et, avec elle, «la conformité à ce qui est rationnellement admissible» selon Robert Martin ${ }^{89}$. Dans l'extrait de Godet des Marais, la citation tronquée semble décréter une impuissance de l'âme face au corps et entraîner une soumission aux pulsions, autrement dit un glissement de la passivité mystique à la passion. Le paradoxe réside dans une forme d'inaction (le "recueillement ») devant le danger des passions. De fait, l'expression de Madame Guyon "Une seule chose le peut faire", équivalant à la négation exceptive en "ne... que», met en scène ce paradoxe du seul recours à l'oraison face à un danger si tangible.

41 Cependant, ce paradoxe n'est plus que formel lorsque «l'épilogue » au sens d'Aristote ou les « justifications » au sens de la Rhétorique à Alexandre sont rétablis. L'expression de la cause en car décrète justement un primat de l'âme sur le corps (« les sens ne peuvent tirer de vigueur que de l'application de l'âme ») en même temps qu'elle refuse de lier logiquement deux notions qui partagent une étymologie : la passivité (mystique) et la 
passion ("Cette vie des sens émeut et irrite la passion, loin de l'éteindre »). Cette expression de la cause est ce qui permet à la figure du paradoxe à double détente d'être formulé, au lieu d'être une contradiction insurmontable comme dans la version tronquée de Des Marais. Bien moins que déclarer une incapacité de l'âme, l'invitation guyonnienne consiste à tourner son âme "au-dedans " plutôt qu' "au-dehors", afin que l'âme, si puissante sur les « sens », les préserve des dangers du monde.

À lire la version des quatre ouvrages de Madame Guyon par Paul Godet des Marais, on est tentée de dire, comme la petite Alice de Lewis Carroll : «Je ne puis croire pareille chose !». En effet, si l'expérience mystique de Madame Guyon assume sans nul doute une expression féminine et charnelle, sa réécriture par l'évêque de Chartres caricature ce mouvement néo-platonicien en hystérie hérétique qui nie les vertus de la charité et de l'espérance. La décontextualisation systématique des paradoxes guyonniens les ampute de leur épilogue, que celui-ci prenne la forme de relatives essentielles, de l'expression de la cause, ou d'un déterminant défini transformé en déterminant possessif.

L'usage du paradoxe conserve son ambivalence antique. Figure discréditée et pourtant pratiquée, il constitue chez Mme de Guyon une ressource argumentative et un instrument pour asseoir ses prises de positions. Mais pour le père Godet des Marais, il est l'instrument de manipulation et d'instrumentalisation dans son travail de sape de l'adversaire. Finalement, paradoxe du paradoxe ou paradoxe au carré, les travers prétendus du paradoxe sont exploités et mis à contribution de différentes manières, nullement censurés de fait.

Un véritable débat théologique n'a donc pas lieu. Emprisonnée sur lettre de cachet le 27 décembre 1695, Madame Guyon est libérée le 24 mars 1703, sur un brancard, sans que l'on ait rien pu prouver contre elle : «Les institutions ecclésiastiques ne pouvaient que réagir devant cet "enthousiasme"; que ce soit une femme qui développe ces thèmes eschatologiques ne pouvait qu'accroître leur méfiance ${ }^{90} »$.

\section{NOTES}

1. L. Carroll, De L'Autre Côté du miroir et ce qu'Alice y trouva [1872], trad. H. Parisot, Paris, Gallimard, Bibliothèque de la Pléiade, 1971, p. 305.

2. Dans Le Banquet, par exemple, le premier éloge d'Éros par Phèdre oppose la " divine vaillance » et la virilité d'Alceste (pourtant une femme, celle d'Admète) et d'Achille à la lâcheté du poète Orphée qualifié d' « efféminé ».

3. Proche de Madame du Châtelet et d'olympe de Gouges, «Voltaire entend cependant préserver la vigueur propre d'une masculinité de la pensée qui appartient aussi à la femme » (Fl. Lotterie, Le Genre des Lumières. Femme et philosophe au XVIII siècle, Paris, Classiques Garnier, 2015, p. 114).

4. T. d'Avila, CEuvres complètes, Desclée de Brouwer [1964], 2007, t. II, p. 1059 : «l'entendement n'agit point ». 
5. J.-J. Surin, Cantiques spirituels de l'amour divin, éd. B. Papàsogli, L. S. Olschki, Florence, 1996, « De l'abandon à la divine providence », p. 62 : «l'esprit se perd » et l'on «ferm[e] les yeux ».

6. S. Houdard, «Le problème de l'écriture et du style mystiques ", Littératures classiques, $\mathrm{n}^{\circ} 50$, 2004, p. 305-318.

7. F. Malaval, "La Plainte de Philotée", La belle ténèbre, Pratique facile pour élever l'âme à la contemplation [1670], éd. M-L. Gondal, Grenoble, J. Millon, 1993, p. 302.

8. La raison n'est plus dépassée ou amuie, elle meurt totalement chez Madame Guyon. Il faut "[...] entrer dans la funeste expérience de toutes les misères [...]. Et c'est ce qui opère véritablement la mort de l'âme [...] il faut passer de longues et effroyables ténèbres [...]. C'est où la raison se perd. [...] C'est donc la perte de cet imperceptible moyen et l'expérience de ses misères qui causent la mort » (Les Torrents, dans Euvres mystiques, éd. D. Tronc, Paris, Honoré Champion, 2008, p. 216).

9. Article «Langage de la mystique ", Dictionnaire de la mystique [Wöterbuch der Mystik, Stuttgart, Alfred Kröner Verlag, 1989], éd. P. Dinzelbacher, Turnhout, Brepols, 1993, p. 477.

10. S. Houdard, op. cit., p. 305-307.

11. Mme Guyon, « À Fénelon, 21 juin ? 1689 », CEuvres mystiques, op. cit., p. 433.

12. L'argument pourrait même être retourné dans la mesure où le paradoxe est, depuis Socrate, ce qui permet de s'élever au-dessus de la doxa pour viser la vérité.

13. H. Bremond, Histoire littéraire du sentiment religieux en France depuis la fin des Guerres de Religion jusqu'à nos jours, 12 volumes, Paris, Bloud \& Gay, 1916-1936, tome II, p. 261-262. Nous soulignons.

14. Il est dépeint par Saint-Simon : Mémoires, éd. Boislisle, Paris, 1879-1928, II p. 207, III p. 14 et sq., IV, p. 83, VII, p. 178-179, VIII p. 77 et sq., XIV p. 118-119 et 382-383, XVII, p. 49, XVIII p. 229, XXVII, p. 55, XXXVII, p. 189.

15. Moyen court et très facile pour l'oraison que tous peuvent pratiquer très aisément... (1685), dans Euvres mystiques, éd. D. Tronc, Paris, Honoré Champion, 2008, p. 63-64.

16. J. Le Brun, "Paul Godet des Marais, évêque de Chartres (1648-1709) », Bulletin de la Société archéologique d'Eure et Loir, $2^{\mathrm{e}}$ trimestre 1965, XXIII, Chartres, 1965, p. 47-78.

17. P. Godet des Marais, Ordonnance et instruction pastorale de Monseigneur l'Évêque de Chartres pour la condamnation des livres intitulés Analysis orationis mentalis \& Moyen court et très-facile de faire oraison \& Règles des associés à l'Enfance de Jésus \& Le Cantique des cantiques de Salomon, interprété selon le sens mystique \& d'un manuscrit qui a pour titre Les Torrents, à Paris, chez Louis Josse, Imprimeur et libraire de Monseigneur l'Archevêque, rue Saint Jacques, à la Couronne d'épines, 1695 [désormais Ordonnance].

18. R. Waldo Emerson, The Journals and Miscellaneous Notebooks, V, 1835-1838, éd. M. M. Sealts Jr., Cambridge, Belkhap Press of Harvard University Press, 1965, p. 5.

19. Voir V. Novarina, "Ouverture", dans J. Beaude, H. Bourgeois, M. Cariou, M. Chevallier, É. Goichot, M.-L. Gondal, H. Hillenaar, J. Le Brun, Cl. Louis-Combet, V. Novarina, I. Noye, P. Pachet, H.-J. Schrader, P. A. Ward, Madame Guyon, Grenoble, Jérôme Millon, 1997.

20. Le Moyen court et autres récits, éd. M.-L. Gondal, Grenoble, Jérôme Millon, 1995, p. 82 : « Dieu détruit, brûle, et purifie » (appendice) et « Le centre a toujours une vertu attractive très forte. Et plus le centre est éminent et spirituel, plus son attrait est violent et impétueux, sans pouvoir être arrêté. »

21. Moyen Court, éd. Gondal, II, 21, p. 603 et 608.

22. B. Forthomme, J. Hatem, Madame Guyon : quiétude d'accélération, Paris, Cariscript, 1997, p. 5.

23. Moyen Court, éd. Gondal, p. 53-63.

24. Les Torrents spirituels. Traité [Rédaction vers 1682, 4 manuscrits de 1698 environ, une publication de la première partie en 1699, puis du texte complet en 1712 en Hollande par Pierre Poiret], dans Euvres mystiques, op. cit., p. 180.

25. Ibid., note 174 de la page 180.

26. Titre du chapitre X du Moyen Court : « De la mortification ». 
27. Moyen Court, éd. Gondal, p. 146 et 153.

28. Voir A. Mezzadri-Guedj, Fénelon, du paradoxe à la répétition. Pur Amour, pur style, Paris, Classiques Garnier, 2020.

29. F. Godefroy, Dictionnaire de l'ancienne langue française du $9^{e}$ au $15^{e}$ siècle, Paris, Wieweg et Bouillon, 1880-1902.

30. P. Richelet, Dictionnaire françois [1679], Genève, V. Miège, 1693, p. 108. La définition du terme est identique dans la première version du dictionnaire de 1679.

31. Ce mouvement est initié dès le début du siècle dans le cadre de querelles linguistiques. Voir S. Dupleix, Liberté de la langue française dans sa pureté, Paris, Denys, Bechet, 1651, p. 70.

32. Voir C. Perelman et L. Olbrechts-Tyteca, Traité de l'argumentation [1958], § 48, Bruxelles, Éd. de l'Université Libre de Bruxelles, 1988, p. 270-282.

33. J.-C. Scaliger, Poetices libri septem, A. Vincentium, Genève, J. Crespin, 1561.

34. L. de Cressolles, Theatrum veterum rhetorum, oratorum, declamatorum quos in Graecia nominabant sophistas, Paris, S. Cramoisy, 1620.

35. R. Bary, La Rhétorique française [1653], nouvelle édition, Paris, P. le Petit, 1659.

36. L. Bourdaloue, Tractatus rhetoricae; La Rhétorique, traduite pour la première fois, conformément au texte latin (manuscrit) de la bibliothèque d'Alençon, Paris, E. Belin, 1864.

37. B. Lamy, La Rhétorique ou L'Art de parler [1675], éd.Ch. Noille-Clauzade, Paris, Honoré Champion, 1998, p. 216-237.

38. Breton, De la rhétorique selon les préceptes d'Aristote, de Cicéron, et de Quintilien. Avec des exemples tirés des auteurs sacrés et profanes tant anciens que modernes. Divisé en trois livres, Paris, Grégoire du Puy, 1703.

39. J. de Jouvancy, L'Élève de rhétorique [Candidatus rhetoricae, 1710], éd. sous la dir. de F. Goyet et D. Denis, Paris, Classiques Garnier, 2010, p. 198-203.

40. R. Rapin, Réflexions sur l'usage de l'éloquence de ce temps [1672] et Réflexions sur la poétique d'Aristote et sur les ouvrages des poètes anciens et modernes [1676], dans Euvres du père Rapin [...], dernière édition augmentée du Poème des jardins, tome second, La Haye, P. Gosse, 1725.

41. C. F. de Vaugelas, [Remarques sur la langue française utiles à ceux qui veulent bien parler et bien écrire, Paris, chez la Veuve Camusat et P. Le Petit, 1647], Remarques, nouvelle édition, comprenant le texte de l'édition originale, des remarques inédites, une clef inédite de Conrart, tous les commentaires du xviie siecle, des notes nouvelles, une introduction et une table analytique des matieres, par A. Chassang, Versailles, Cerf et fils, 1880, I, p. 193.

42. J. B. L. Crevier, Rhétorique françoise, Paris, Saillant et Desaint, 1765, p. 255-256.

43. La fin du paragraphe cicéronien résout l'attente : « c'est en Macédoine, au camp de Pompée, au cœur même du parti qui l'avait ignominieusement rejeté ». N. Caussin, Eloquentiae sacrae et

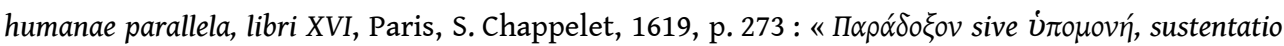
vel inopinatum. Hoc schema suspendit sensum, deinde subjicit aliquid eo, contra exspectationem auditoris, sive majus sive minus, et ideo sustentatio vel inopinatum dicitur. Cicero pro Ligario: Hinc prohibitus non ad Caesarem; ne iratus, non ad domum, ne iners, non aliquam in regionem, ne condemnare causam illam, quam secutus et caetera. » Nous traduisons ; la traduction du Pour Ligarius, 27, est celle de Marcel Lob dans son édition, Paris, Les Belles Lettres, C. U. F., 1968.

44. R. Lulle, "Traité de toutes les figures de rhétorique, suivant l'ordre de l'alphabet", La Clavicule, ou la Science de Raymond Lulle, avec toutes les figures de rhétorique par le sieur Jacob et la Vie du même Raymond Lulle, par M. Colletet [1647], Paris, M. Bobin, 1655, p. 187-188.

45. Voir M. Bonhomme, Les figures clés du discours, Paris, Le Seuil, 1998, p. 81 ; et du même auteur, Pragmatique des figures du discours, Paris, Honoré Champion, 2005, p. 56.

46. D. Bouhours, La manière de bien penser dans les Ouvrages d'esprit, Paris, Vve de S. MabreCramoisy, 1688, p. 67.

47. Mme Guyon, La Vie par elle-même et autres écrits biographiques, Paris, Honoré Champion, 2001, p. 763. 
48. J. de Champeygnac, Sommaire des quatre parties de la philosophie: logique, éthique, physique et métaphysiques, Paris, F. Bourriquant, 1607.

49. S. Dupleix, La Logique, ou Art de discourir et raisonner [1604], Paris, Fayard, 1984.

50. A. Geulincx, « De Sophismate », Logica fundamentis suis, Lyon, H. Verbiest, 1662, p. 516.

51. J. Clauberg, [Claubergii Logica vetus et nova, quadripartita, modum inveniendae ac tradendae veritatis, in Genesi simul et analysi, facili methodo exhibens, Amstelaedami, ex officina Elzeviriana, 1658, seconde édition après celle de 1654], Logique ancienne et nouvelle, II, 12 (sommaire du chapitre), éd. et trad. J. Lagrée et G. Coqui, Paris, Vrin (Bibliothèque des textes philosophiques), 2007, p. 157. Nous soulignons.

52. Ibid., §82, p.159-160. «Interprétations complexes» traduit «in complexis interpretandis» (sous-entendu «animi notionibus»), par opposition à «in simplicibus animi notionibus exponendis " (" dans l'exposition des notions simples de l'âme », trad. Lagrée et Coqui).

53. Dans le Gorgias, à l'opinion commune de Polos, Socrate oppose le paradoxe que « commettre l'injustice [est] pire que la subir» (Platon, Gorgias 473b-474a, trad. M. Canto, Paris, Garnier Flammarion, 1987, p. 183-185).

54. A. Arnauld et P. Nicole, La Logique ou l'art de penser [1662], Paris, Honoré Champion, 2011, p. 630 .

55. Ibid., p. 549.

56. Il s'agit donc d'une précision de la définition comme « contre-vérité » de M. Bonhomme dans son relevé de figures du discours : "[l]'énoncé 'Le soleil se sert très frais' exprime une contrevérité à propos de la réalité solaire, mettant en évidence le schème du paradoxe » (M. Bonhomme, Pragmatique des figures du discours, Paris, Honoré Champion, 2005, p. 56).

57. M. Carel et O. Ducrot, « Les propriétés linguistiques [...] », op. cit., p. 27.

58. Aristote, La Rhétorique, II, 23, 1400a26-28, éd. et trad. M. Dufour, Paris, « Les Belles Lettres ", 1991, II, p. 125.

59. Mme Guyon, Correspondance, Tome II, éd. D. Tronc, Paris, Honoré Champion, 2004, p. 505 et 507.

60. La plus grande coupure de Godet des Marais a lieu dans Les Torrents entre les paragraphes 13 et 21 de la première partie, chapitre VII section I « Troisième degré de la voie passive en foi ». Les cinq points de suspension du texte de l'Ordonnance correspondent à un saut du § 13 au $\S 21$, soit plusieurs pages.

61. Nous référençons les extraits de l'Ordonnance à l'endroit où ils prennent fin; ils peuvent commencer en amont, compte tenu des coupures; les numéros des paragraphes sont indiqués entre crochets.

62. Concernant le Moyen court, les chapitres I « Tous peuvent faire oraison », II « Manière de faire oraison», III «Pour ceux qui ne savent pas lire», IV «Second degré d'oraison [oraison de simplicité] », V « Des sécheresses », VII « De la souffrance », VIII « Des mystères », IX « De la vertu», XI «De la conversion» et XII «De l'oraison de simple présence de Dieu», XIV «Du silence intérieur ", XVI « De la lecture et des prières vocales », XVII « Des demandes », XVIII « Des défauts », XIX « Des distractions et tentations », XX « De la prière », XXII « Des actes intérieurs » et XXIII «Avertissements aux pasteurs et aux prédicateurs» ne font l'objet d'aucune citation. Dans Les Torrents, il en va de même des chapitres I «Divers retours de l'âme à Dieu, II «Voie active de la méditation », V «Imperfections de ce premier degré. Sécheresses » et VI « Deuxième degré de la voie passive en foi » de la première partie.

63. V. Carraud, « Deux degrés de néant : les fictions impossibles des nouveaux mystiques », Revue Bossuet, 2011/2, p. 71-97.

64. Moyen Court, éd. Gondal, p. 98.

65. P. Godet des Marais, Ordonnance, op. cit., p. 24.

66. G. Moignet, Systématique de la langue française, Paris, Klincksieck, 1981, p. 205. 
67. Réponse de Monseigneur l'archevêque de Cambrai à l'écrit de monseigneur l'évêque de Meaux intitulé relation sur le quiétisme, Euvres complètes de Fénelon, édition dite de Paris, de Saint-Sulpice, ou des quatre éditeurs (Leroux, Gaume [Paris], Lefort [Lille], Outhenin-Chalandre [Belfort]), dix tomes, 1848-1852, " par les soins de MM. Gosselin et Caron », tome III, p. 24. Nous soulignons.

68. Voir la Première épître aux Corinthiens de saint Paul.

69. Mme Guyon, Moyen court, éd. Gondal, première partie, chapitre IV «Voie passive en foi, premier degré », p. 176.

70. P. Godet des Marais, Ordonnance, op. cit., p. 19.

71. M. Riegel, J.-C. Pellat et R. Rioul, Grammaire Méthodique du Français, Paris, P.U.F, «Quadrige Manuels ", 2009, p. 806-807.

72. P. Godet des Marais, Ordonnance, op. cit., p. 24.

73. P. Godet des Marais, Ordonnance, op. cit., p. 25.

74. Citation biblique : Épitre aux Romains, $4,18$.

75. Citation biblique : Évangile selon saint Matthieu, 6, 34.

76. Citation biblique : Prophètes, 3, 6.

77. Citation biblique : Prophètes, $16,3$.

78. Citation biblique : Psaumes, $37,5$.

79. Moyen Court, éd. Gondal, p. 84. La note 88 mentionne l'utilisation de ce passage par Godet des Marais.

80. Citation biblique : Épitre aux Romains, 4, 18.

81. Citation biblique : Épitre aux Romains, $4,18$.

82. P. Godet des Marais, Ordonnance, op. cit., p. 11.

83. Aristote, La Rhétorique, II, 21, 1394b7-11 (voir aussi 27-34), op. cit., II, p. 107-108.

84. Ps. Aristote, Rhétorique à Alexandre [IV siècle av. J.-C.], 1430b, éd. et trad. P. Chiron, Paris, Les Belles Lettres, 2002, p. 47-48.

85. P. Godet des Marais, Ordonnance, op. cit., p. 4.

86. Les Torrents, « Voie passive et Lumière ", op. cit., I, 3, p. 164.

87. P. Godet des Marais, Ordonnance, op. cit., p. 25.

88. J.-C. Anscombre, «Étude pragmatique des connecteurs 'car', 'parce que', 'puisque' et 'décidément' ", Cahiers de Grammaire de Toulouse, 1984, nº 8, p. 49.

89. R. Martin, «Le mot puisque : notions d'adverbe de phrase et de présupposition sémantique ", Studia neophilologica, XLC, 1973, p. 114.

90. J. Le Brun, au sujet des écrits de Madame Guyon dans La Jouissance et le trouble, Recherches sur la littérature chrétienne de l'âge classique, Genève, Droz, 2004, p. 297.

\section{AUTEUR}

\section{AGATHE MEZZADRI-GUEDJ}

Lycée Jules Michelet 